\section{AUTOIMMUNITY \\ T1DM AND THE GUT MICROBIOME}

The genetic contribution to development of type 1 diabetes mellitus (T1DM) is well established. However, a rapid increase in prevalence of the disease over the past few decades and the finding that only a small portion of children who carry predisposing HLA risk alleles develop T1DM suggests that the aetiology of the disease also has a considerable nongenetic component. A new study shows that changes in gut microbiome of infants with genetic susceptibility to T1DM occur as early as 1 year prior to disease onset, which supports a hypothesis that the microbiome represents a considerable nongenetic contributor to the disease and could be a viable therapeutic target.

The associations between the gut microbiome and systemic immunity, in particular the development of autoimmunity, have largely been evaluated in studies in animals or in clinical studies with only small cohorts of participants. A team of researchers from the Broad Institute of MIT and Harvard University in Cambridge MA, USA, have conducted what they believe is the largest longitudinal study of the human microbiome to date. They analyzed the microbiomes of 33 HLA-matched infants, collecting samples at monthly intervals from birth until 3 years of age. Study participants were selected on the basis of having HLA risk alleles associated with T1DM. Of the 33 infants, 11 seroconverted to serum autoantibody positivity (thus termed 'seroconverters') during the course of the study. Moreover, four of the seroconverters developed T1DM.

The taxonomic composition of the microbiomes varied widely between individuals, as well as within the same individual, over the duration of the study period; however, the major metabolic components of the microbiomes remained fairly stable. Importantly, unique changes to the microbiomes were shared among the seroconverters, including a sharp decrease in microbiome diversity, an increase in pathobionts and a decrease in bacteria that produce short-chain fatty acids.

The group plans to continue their investigations in larger cohorts. "The gut microbiome might be a new avenue by which to approach prevention, diagnosis and/or treatment of T1DM," says Aleksandar Kostic, lead author of the study.

Jennifer Sargent

Original article Kostic, A. D. et al. The dynamics of the human infant gut microbiome in development and in the progression toward type 1 diabetes. Cell Host Microbe 17, 260-273 (2015) 